\title{
Curative Effect and Safety of Tolvaptan Combined with Traditional Diuretics in Treatment of Patients with Cirrhotic Ascites and Relevant Research on its Dose: A Systematic Review with Meta-Analysis of Randomized Controlled Trials
}

Xin Li

Lanzhou University

Shidong Liu

Lanzhou University

Hui Zhang

Lanzhou University

Fuxiang Liang

Lanzhou University

Long Miao

Lanzhou University

Hao Xu

Lanzhou University

Zhenjie Zhao

Lanzhou University

Wence Zhou ( $\sim$ zhouwenceldyy@163.com)

Lanzhou University https://orcid.org/0000-0001-9587-5798

Research article

Keywords: Tolvaptan; Cirrhosis of the liver; Ascites; Diuretics; Randomized controlled trials.

Posted Date: April 24th, 2020

DOI: https://doi.org/10.21203/rs.3.rs-17637/v2

License: (c) (i) This work is licensed under a Creative Commons Attribution 4.0 International License. Read Full License 


\section{Abstract}

Background: Tolvaptan is a receptor antagonist of highly selective vasopressin V2, and it can promote excretion of water without electrolyte.

Aims: To evaluated curative effect and safety of Tolvaptan in treatment of cirrhotic ascites, and its relationship with drug dose.

Methods: Computer retrieval of PubMed, EMbase and The Cochrane Library was carried out to search the clinical trials of cirrhotic ascites treatment by Tolvaptan. The time limit of retrieval is from the database setup to May 31 , 2019. 2 researchers independently screened studies, extracted the data and crosschecked. RevMan 5.3 software was used for Meta-analysis.

Results: Finally, 6 articles and 848 patients were included in the study. Meta-analysis indicates that after the intervention for 7 days, serum sodium ion concentration of the combined diuretic group obviously increased, compared with the traditional diuretic group $(\mathrm{WMD}=2.92 \mathrm{mmol} / \mathrm{L}, 95 \% \mathrm{Cl}[2.15,3.70], \mathrm{P}<0.001)$. In addition, Tolvaptan also can decrease patients' ascites amount through increasing liquid discharge. However, the drug dose has no obvious correlation with the reduction degree of ascites amount. The rise of blood uric acid is the major adverse event in the treatment of cirrhotic ascites patients by Tolvaptan $(\mathrm{OR}=6.01,95 \% \mathrm{Cl}[1.11,32.56], \mathrm{P}=0.04)$. When the dose of Tolvaptan rose to $15 \mathrm{mg}$ or $30 \mathrm{mg}$, the total incidence of adverse events was obviously higher than it of traditional diuretic group.

Conclusion: Tolvaptan combined with traditional diuretics has good curative effect for the patients with cirrhotic ascites. As its adverse reactions, the recommended dose of Tolvaptan is $7.5 \mathrm{mg}$ in the combined therapy.

\section{Background}

Ascites and hyponatremia are the major complications in patients with liver cirrhosis. Within 10 years, $60 \%$ of patients with decompensated cirrhosis will develop ascites, with a higher proportion of patients with ascites in the decompensatory phase [1]. This also leads to a variety of subjective symptoms in patients with cirrhosis, thus reducing their quality of life [2]. The one-year survival rate of the patients in decompensated cirrhosis with ascites and hyponatremia is only $60 \%$ [3]. Liver transplantation is the only way to cure decompensation of liver cirrhosis, but most patients still adopt conservative medication and palliative surgery. Traditional diuretics represented by furosemide are the main drugs for the treatment of cirrhotic ascites [4].

However, traditional diuretics will cause hyponatremia and about $15 \%$ of patients have no response to traditional diuretics, which is a major problem in the treatment of cirrhotic ascites [5-6]. Unlike traditional diuretics, Tolvaptan is a receptor antagonist of highly selective vasopressin V2 that promotes excretion of water without electrolyte. Besides, it neither influences in vivo electrolyte content except for serum sodium ion, nor easily results in kidney failure [7]. Tolvaptan has been approved by many countries around the world for the treatment of autosomal dominant polycystic kidney disease, cardiac failure and hyponatremia [8-9]. However, most studies on the efficacy of torvaptan in the treatment of cirrhotic ascites are retrospective or observational, and the safety of Tolvaptan in treatment of cirrhotic ascites is still questioned, including the incidence of liver injury and other adverse reactions. Thus, Tolvaptan is only approved to treat cirrhotic ascites in a minority of regions including Japan $[8,10]$.

Currently, there are only a few meta-analyses for vaptans drugs (including Tolvaptan, Satavaptan and Lixivaptan), and few results on Tolvaptan, not to mention a comprehensive analysis on the efficacy and safety of Tolvaptan [11, 
12]. A recent meta analysis for retrospective cohort studies of Tolvaptan reported total survival after application, but no detailed data were available on treatment outcomes and complications [13]. Moreover, we found a meta analysis for Tolvaptan (in Chinese) [14], but most (5/8) of the studies included were single-center clinical trials published in Chinese journals. These trials were not registered $\Downarrow$ nor do they describe explicit hidden allocation method and detailed experimental process, so we consider the results of this paper to be suspect.

To evaluate curative effect and safety of Tolvaptan in treatment of cirrhotic ascites, and its relationship with drug dose, this study reviews the randomized controlled trial (RCT) of Tolvaptan in the treatment of cirrhotic ascites.

\section{Method}

\section{Data and method}

The Meta-analysis was performed on the basis of systematic reviews and Meta-analysis of preferred reporting projects and was prospectively registered with the PROSPERO database (ID: CRD42019143480).

\section{Type of study}

RCT study on Tolvaptan combined with traditional diuretics (loop diuretic and/or anti-aldosterone agent) and conventional diuretics (with/without placebo) in treatment of liver cirrhosis with ascites.

\section{Object of study}

The patients diagnosed with ascites, only caused by liver cirrhosis and received the treatment by traditional diuretics. Intervention measure

The patients received the treatment by Tolvaptan combined with traditional diuretics or pure traditional diuretics (with/without placebo).

Treatment with a combination of traditional diuretics or traditional diuretics alone (with/without placebo) was given.

\section{Outcome indexes}

Major indicator of curative effect: the changes of serum sodium ion. Minor indicator of curative effect: changes in ascites (weight, abdominal girth and ascites amount) and urine volume changes on the first day after medication. Major safety indexes: total incidence of adverse events. Minor safety indexes: total incidence of severe adverse events (including death or the events endangering life, continuous or severe disability, and the increase in the times of hospitalization) and incidence of various common adverse events (increase of blood uric acid, frequency of urination, thirst, constipation, hepatic encephalopathy, diarrhea, fever, insomnia, renal function damage and hypokalemia). Moreover, the changes of the above indexes in different doses were evaluated.

\section{Exclusion criteria}

(1) Non-RCT study; (2) articles published repeatedly; (3) articles from which the data cannot be extracted.

\section{Study retrieval strategy}

Computer retrieval of PubMed, EMbase and The Cochrane Library was carried out to search the clinical trials of cirrhotic ascites treatment by Tolvaptan. Retrieval time limit from database Settings to August 31, 2019 Meanwhile, 
supplementary retrieval of research data was conducted, including WHO clinical trial registry platform (http://apps.who.int/trialsearch), Chinese clinical trial registry platform (http://www.chictr.org.cn) and American clinical trial registry library (https://clinicaltrials.gov). Retrieval time limit from database Settings to August 31, 2019. Furthermore, the references included in the papers were traced to supplement and gain relevant studies. The subject terms and free words were combined for the retrieval. The search terms contains Tolvaptan, Samsca, Jinarc, Jynarque, OPC41061, OPC 41061, OPC-41061, and Ascites. The search algorithm is ((Tolvaptan or Samsca or Jinarc or Jynarque or OPC41061 or OPC 41061 or OPC-41061) and Ascites). Literature retrieval is not restricted by language.

\section{Study screening and data extraction}

2 researchers independently screened studies, extracted the data and cross-analyzed them. Differences should be resolved through discussion and consultation. During the screening period, the title was read for the first time. After excluding apparently unrelated studies, the abstract and full text are further read to determine whether the article was included. If necessary, the original authors could be contacted by email and telephone for information that is uncertain but important in this study. The extracted data include (1) research features, including the first author, publication year, country, random method, blind method and duration of mediation (research period); (2) general features of patients, including the number, age and gender in each research group; (3) therapeutic evaluation indexes, including the changes of serum sodium ion, bodyweight abdominal girth, and ascites amount as well as the change in urine volume on the first day of medication; (4) safety evaluation indexes, including total incidence of adverse events, total incidence of severe adverse events, and the incidence of various common adverse events (diarrhea, frequency of urination, fever, hepatic encephalopathy, insomnia, increase of blood uric acid, and hypokalemia); (6) dose-related safety evaluation indexes and therapeutic evaluation indexes: the occurrence rates of safety evaluation indexes and therapeutic evaluation indexes under different dose were collected.

\section{Methodological quality evaluation}

The two researchers assessed the risk of bias within the study. According to the Cochrane Handbook for Systematic Reviews of Interventions, 2011)[15], the evaluation indexes include: $\nabla$ selection bias, random sequence generation and

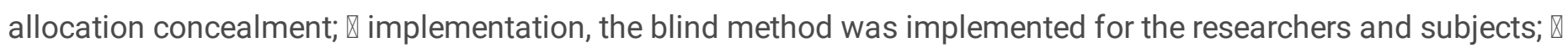
measurement, evaluation of research results with the blind method; $\nabla$ follow-up visit, integrity of result data; $\nabla$ reporting, selective reporting of research results; $\nabla$ others. If the above indexes are of low risks, the study is evaluated as low bias risk, and other results are considered to have high bias risk.

\section{Statistical method}

RevMan 5.3 software (The Nordic Cochrane Centre, Copenhagen, Denmark) was used for Meta-analysis. The weighted mean difference (WMD) was used as the effect quantity in the continuous outcome data. For the result data of dichotomy, the odds ratio (OR) was used as the effect size. The point estimate and 95\% credibility interval (95\% Cl) were given for each effect size. The heterogeneity of research results was analyzed by $\chi^{2}$ test, and the heterogeneity degree was quantitatively determined by combining $\mathrm{I}^{2}$. $\mathrm{P}<0.10$ or $\mathrm{I}^{2}>50 \%$ were considered as significant heterogeneity. The publication bias of all end points was first judged by the funnel plot. Then, Egger test was adopted for quantitative assessment. $\mathrm{P}<0.05$ means the difference is statistically significant.

\section{Results}

\section{Study screening and bias analysis}


Based on the above search method, a total of 220 qualified literatures were found.. A total of 46 articles were retrieved by reading and removing abstracts of the studies that did not meet the inclusion criteria. They were downloaded, read and further assessed. Among them, 32 references are non-RCT researches; There were no objective indexes in 6 literatures; two references are of abstract form, without objective outcome indexes. The detailed screening process is shown in Fig 1. Finally, 6 articles [16-21] were eventually included, with a total of 848 patients (table 1). Among them, 530 patients received Tolvaptan treatment, and there were 318 persons in the control group.

3 studies do not explain the detailed and specific randomization methods, and the other 3 studies interpret the randomization methods, including random number generated by the computer and center randomization. 4 articles describe the specific allocation scheme hiding method. 5 studies were double-blind and 1 was triple-blind. 5 articles report the changes in serum sodium ions, and 5 articles report the overall incidence of adverse events. All articles describe specific follow-ups, including the number and reasons of patients without follow-up visit. All studies are registered in the clinical database. The risk of bias in the study is shown in Supplement Fig 1.

\section{Curative effect}

To evaluate the curative effect, the changes in serum sodium concentration, average body weight, average waistline and average ascites amount and changes in urine volume 7 days after intervention were compared between the two groups on the first day of intervention and the last day.

5 studies report the change of serum sodium concentration (including 758 patients) [16-18, 20-21]. Meta analysis indicates that after 7 days of intervention, the serum sodium concentration of the combined diuretic group was significantly higher than that of the conventional diuretic group $(\mathrm{WMD}=2.92 \mathrm{mmol} / \mathrm{L}, 95 \% \mathrm{Cl}[2.15,3.70], \mathrm{P}<0.001, \mathrm{Fig}$ $2 D)$. There was a significant heterogeneity $\left(I^{2}=61 \%\right.$, Fig $\left.2 D\right)$, sensitivity analysis showed that $I^{2}$ was $4 \%$ when $/$ sao 2014 was removed (Supplement Fig 2A).

In terms of the minor indexes, the changes in the average weight, average waistline and average ascites amount are reported in 4 studies (499 patients) [17-20], 3 studies (440 patients) [17-18, 20], and 2 studies (216 patients) [17, 19], respectively. Four studies (498 patients) reported changes in urine volume on the first day of intervention and on the last day of intervention [16-17, 19-21]. After the intervention for 7 days, the reduction of average weight $(\mathrm{WMD}=-1.51 \mathrm{Kg}, 95 \% \mathrm{Cl}[-1.86,-1.16], \mathrm{P}<0.001$, Fig 2A), average abdominal girth (WMD $=-2.05 \mathrm{~cm}, 95 \% \mathrm{Cl}[-2.67,-1.43]$, $\mathrm{P}<0.001$, Fig 2B), and the average ascites amount $(\mathrm{WMD}=-293.43 \mathrm{~mL}, 95 \% \mathrm{Cl}[-502.53,-84.34], \mathrm{P}=0.006$, Fig 2C) of combined diuretic group was obviously better than that of traditional diuretic group. Besides, the increase in urine volume in the combined diuretic group on day 1 was greater than that in the conventional diuretic group $(\mathrm{WMD}=1.09 \mathrm{~L}$, $95 \% \mathrm{Cl}[0.85,1.33], \mathrm{P}<0.001$, Fig 2E).

In the subgroup analysis, with the increase of Tolvaptan rose $(7.5 \mathrm{mg}-15 \mathrm{mg}-30 \mathrm{mg})$, the serum sodium concentration in the combination diuretic group also increased significantly (Fig 3A). However, the increase in Tolvaptan dose did not cause significant changes in body weight (Fig 3B) and abdominal circumference (Fig 3C). In general, the rise of Tolvaptan dose resulted in limited improvement of curative effect for patients.

\section{Safety}

In safety evaluation, total incidence of adverse events, total incidence of severe adverse events, and the incidence of various common adverse events (diarrhea, frequency of urination, fever, hepatic encephalopathy, insomnia, increase of blood uric acid, and hypokalemia) in the both groups were mainly compared during the intervention. And, the occurrence rates of total adverse events, diarrhea and frequency of urination in different dose groups were evaluated. 
5 studies report the total incidence of adverse events (including 620 patients) during the intervention [16-20]. Mate analysis result shows that the total incidence of adverse events was obviously higher than that of traditional diuretic group $(\mathrm{OR}=1.98,95 \% \mathrm{Cl}[1.11,3.52], \mathrm{P}=0.02$, Fig $5 \mathrm{~A})$. There was a significant heterogeneity $\left(\mathrm{I}^{2}=50 \%\right.$, Fig $\left.4 \mathrm{~A}\right)$, sensitivity analysis showed that $\mathrm{I}^{2}$ was $0 \%$ when Haruki 2017 was removed (Supplement Fig. 1B).

In the subgroup analysis of adverse events, we found no statistical difference in the total incidence of adverse events between the combined diuretic group and the conventional diuretic group when the daily dose of the combined diuretic group was maintained at $7.5 \mathrm{mg}(\mathrm{OR}=1.28,95 \% \mathrm{Cl}[0.66,2.47], \mathrm{P}=0.46$, Fig $4 \mathrm{C} 7.5 \mathrm{mg})$. When the daily dose of Tolvaptan rose to $15 \mathrm{mg}(\mathrm{OR}=2.99,95 \% \mathrm{Cl}[1.03,8.69]$, $\mathrm{P}=0.04$, Fig $4 \mathrm{C})$ or $30 \mathrm{mg}(\mathrm{OR}=3.54,95 \% \mathrm{Cl}[1.73,7.24], \mathrm{P}<0.001$, Fig $4 \mathrm{C}$ ), the total incidence of adverse events in the combined diuretic group was significantly higher than that of traditional diuretic group. In terms of the incidence of severe adverse events, there was no statistical difference between the two groups $(\mathrm{OR}=1.05,95 \% \mathrm{Cl}[0.60,1.84], \mathrm{P}=0.88$, Fig 4B).

Common adverse events include the increase of blood uric acid, frequent urination, thirst, constipation, hepatic encephalopathy, diarrhea, fever, insomnia, renal function damage and hypokalemia. The detailed Mate analysis results are shown in Supplement Table 1. 2 articles (282 patients) $[18,20]$ report the occurrence rate of blood uric acid rise in the intervention process. The combined diuretic group was significantly higher than that of traditional diuretic group $(\mathrm{OR}=6.01,95 \% \mathrm{Cl}[1.11,32.56], \mathrm{P}=0.04$, Fig $5 \mathrm{~A})$. Although the frequency of urination and the incidence of thirst were not statistically different between the two groups (Supplement Table 1), the subgroup analysis shows that when the daily dose of Tolvaptan reached $15 \mathrm{mg}$ or $30 \mathrm{mg}$, the occurrence rates of frequency of thirst (Fig 5B) and urination (Fig $5 \mathrm{C}$ ) in the combined diuretic group were obviously higher than those of traditional diuretic group.

\section{Discussions}

In this study, compared with the traditional diuretic group, the combination of Tolvaptan and traditional diuretics can effectively increase the serum sodium level of patients with cirrhotic ascites. As the dose increased, the increase degree of serum sodium level also rises. In addition, Tolvaptan also can decrease patients' ascites amount (weight, abdominal girth and ascites amount decrease, while the urine volume on the first day increases) through increasing liquid discharge. However, there was no significant correlation between drug dosage and the degree of reduction of abdominal water volume. Meanwhile, the rise of blood uric acid is the major adverse event in the treatment of cirrhotic ascites patients by Tolvaptan combined with traditional diuretics. The rise of Tolvaptan dose did not increase the occurrence rates of severe adverse events and various common adverse events. When the dose of Tolvaptan was controlled at $7.5 \mathrm{mg}$, the total incidences of adverse events in both groups was not abnormal. However, when the dose of Tolvaptan rose to $15 \mathrm{mg}$ or $30 \mathrm{mg}$, the total incidence of adverse events, and the occurrence rates of frequency of urination and thirst were obviously higher than those of traditional diuretic group.

Although the meta analysis of changes of overall serum sodium in our study showed high heterogeneity, the sensitivity analysis found that it was mainly caused by Isao 2014, which focused only on 7.5mg Tolvaptan, while changes of overall serum sodium combined the results of $7.5-30 \mathrm{mg}$ Tolvaptan in the other four studies. The results of subgroup analysis can also explain this high heterogeneity, that is, 1 sao 2014 did not cause heterogeneity at $7.5 \mathrm{mg}$ tolvaptan. Due to its low therapeutic dose, the incidence of total adverse events was reduced, resulting in high heterogeneity in meta analysis of total adverse events.

The common complications of liver cirrhosis include refractory ascites, hyponatremia and hepatic encephalopathy, etc. Especially when the above complications happen to the patients with liver cirrhosis, the death rate will also increase greatly [22]. Tolvaptan is a drug approved by FDA for the treatment of hyponatremia. But multiple countries 
including China still have not approved it to treat cirrhotic ascites. At present, there are only 6 RCT studies on the patients with cirrhotic ascites who are treated by Tolvaptan [16-21]. The curative effect and safety of Tolvaptan in treatment of cirrhotic ascites are still unclear, and there is conclusive evidence that it is not recommended. Moreover, hyponatremia caused by traditional diuretics are also denounced all the time. Some studies also verify that the patients with cirrhotic ascites combined with hyponatremia are closely associated with high mortality [23-24]. Thus, serum sodium level is an important index which cannot be ignored in treatment of cirrhotic ascites by diuretics. The data from this study showed that the application of Tolvaptan significantly improved hyponatremia in patients. Besides, with the increase of Tolvaptan dose (7.5-30mg), the increase degree of patients' serum sodium level also rises.

At the same time, after the use of traditional diuretics, the diuresis effect is not obvious (refractory ascites), which is another problem in the treatment of cirrhotic [25-26]. In particular, inappetence, abdominal distension, dyspnea and other symptoms resulting from lots of ascites seriously affect patients' daily life [2]. So, for the patients with cirrhotic ascites, Tolvaptan combined with traditional diuretics is considered to be a new target for cirrhotic ascites treatment, and its liquid discharge curative effect receives much attention. The results of this study show that compared with pure use of traditional diuretics, Tolvaptan combined with traditional diuretics can significantly increase patients' urine volume (liquid discharge) to reduce ascites amount, indicating that the combined therapy has a better effect on reduction of ascites. In particular, we should encourage the use of it to treat the patients with refractory ascites. However, in terms of reducing the amount, the increase in daily dose of Tolvaptan fails to improve curative effect.

In addition to the good curative effect, complications caused by torvatan are also of great concern. Existing RCT researches report the daily application of low-dose Tolvaptan $(7.5 \mathrm{mg}$ or $15 \mathrm{mg}$ ) promotes 6-month survival rate of patients with cirrhotic ascites [21], and a latest meta analysis also reported that Tolvaptan could significantly improve overall survival rate[13]. However, the concrete complications in the use have not been confirmed by a large number of data. This study demonstrates that it is safe when the daily dose of Tolvaptan is maintained at 7.5mg. The total incidence of adverse events, the incidence of severe adverse events and the incidence of various complications excluding the rise of blood uric acid have no significant differences with those of traditional diuretic group. When the daily dose of Tolvaptan exceeds $15 \mathrm{mg}$, the total incidence of adverse events is obviously higher than that of traditional diuretic group. Especially, the occurrence rates of frequency of urination and thirst rise obviously. Although a previous meta analysis reported that Tolvaptan did not affect mortality and complications in patients with cirrhosis[11] and our study showed that the incidence of severe adverse events has no obvious difference with that of traditional diuretic group, it should be more prudent to apply high-dose Tolvaptan combined with traditional diuretics in treatment of patients with cirrhotic ascites. Furthermore, regardless of Tolvaptan dose, the rise of blood uric acid is the major complication of Tolvaptan combined with traditional diuretics. The specific mechanism still remains to be further demonstrated.

Early meta studies for Vaptan drugs (including Tolvaptan, Satavaptan and Lixivaptan) reported that Vaptan drugs was not associated with prolonged survival in patients with cirrhosis [11-12]. In contrast, a recent meta analysis based on retrospective studies showed that Tolvaptan significantly improves overall survival in patients with cirrhosis and refractory ascites, but this paper has not a high level of evidence [13]. Given the good efficacy and safety of 7.5mg Tolvaptan demonstrated in this study, more RCT studies to study Tolvaptan and survival rates in patients with cirrhotic ascites should be performed to support the prognostic role of tolvaptan.

The limitations of this study are mainly as follows: 1 . Currently, there are still few RCT studies on the treatment of cirrhotic ascites with Tolvaptan, and indexes of each study are not completely consistent; 2 . Some studies did not 
research the relationship between dose and efficacy or safety, so the sample size of dose-related studies was small. In the future, we expect to conduct a larger sample of RCT study on the treatment of cirrhotic ascites with Tolvaptan.

Tolvaptan combined with traditional diuretics is effective in the treatment of cirrhotic ascites. When the dose of Tolvaptan is low $(7.5 \mathrm{mg})$, it is safe. However, from the safety results of high dose use $(\geq 15 \mathrm{mg})$ and the results of dose-related studies, it can be seen that high dose of Tolvaptan does not bring better efficacy, but causes the higher incidence of adverse events. In combination therapy, the recommended dose of Tolvaptan is $7.5 \mathrm{mg}$. In patients who did not respond to $7.5 \mathrm{mg}$ of Tolvaptan, the data do not support application of higher dose Tolvaptan. Besides, it is required to pay close attention to the changes of patients' blood uric acid in the drug use.

\section{Conclusions}

Tolvaptan combined with traditional diuretics is effective in the treatment of cirrhotic ascites. Due to its adverse effects, the recommended dose of Tolvaptan is $7.5 \mathrm{mg}$ in the combined therapy. Changes in serum uric acid should be monitored throughout the course of treatment whether the Tolvaptan dose is elevated.

\section{Abbreviations}

RCT: randomized controlled trial; WMD: weighted mean difference; OR: odds ratio; 95\% Cl: 95\% credibility interval.

\section{Declarations}

Ethics approval and consent to participate: Not applicable.

Consent for publication: The manuscript is approved by all authors for publication. We would like to declare that the work described was original research that has not been published previously, and not under consideration for publication elsewhere, in whole or in part. All the authors listed have approved the manuscript that is enclosed.

Availability of data and materials: We have uploaded relevant meta-analysis data to the submission system as a supplementary file.

\section{Competing interests: None.}

Funding: None.

\section{Authors' contributions:}

$\mathrm{XL}$ was responsible for data extraction, data processing and original manuscript writing. SL was responsible for data extraction and the registration of research scheme. $\mathrm{HZ}$ was responsible for methodology guidance. FL was responsible for the management of original document screening. LM was responsible for the review and revision of draft. HX and ZZ were responsible for the screening of original document. WZ was responsible for research design and management. All authors read and approved the final manuscript.

Acknowledgments: None.

\section{References}

[1] Pere Ginés, Quintero E, Arroyo V, et al. Compensated cirrhosis: Natural history and prognostic factors. Hepatology 1987; 7:122-128. 
[2] Solà E, Watson H, Graupera I, et al. Factors related to quality of life in patients with cirrhosis and ascites: Relevance of serum sodium concentration and leg edema. Journal of Hepatology 2012; 57:1199-1206.

[3] Yu C, Sharma N, Saab S. Hyponatremia: clinical associations, prognosis, and treatment in cirrhosis. Experimental \& Clinical Transplantation 2013; 11:3-11.

[4] European Association for the Study of the Liver. EASL clinical practice guidelines on the management of ascites, spontaneous bacterial peritonitis, and hepatorenal syndrome in cirrhosis. Journal of Hepatology 2010; 53:397-417

[5] Leiva JG, Salgado JM, Estradas J, et al. Pathophysiology of ascites and dilutional hyponatremia: Contemporary use of aquaretic agents. Annals of Hepatology 2007; 6:214.

[6] Salerno F, Calogero Cammà, Enea M, et al. Transjugular Intrahepatic Portosystemic Shunt for Refractory Ascites: A Meta-analysis of Individual Patient Data. Gastroenterology 2007; 133:825-834.

[7] Josiassen RC, Curtis J, Filmyer DM, et al. Tolvaptan: a new tool for the effective treatment of hyponatremia in psychotic disorders. Expert Opinion on Pharmacotherapy 2010; 11:637-648.

[8] Schrier RW, Gross P, Gheorghiade M, et al. SALT Investigators.Tolvaptan, a selective oral vasopressin V2- receptor antagonist, for hyponatremia. New England Journal of Medicine 2006; 355:2099-112.

[9] Matsuzaki M, Hori M, Izumi T, et al. Efficacy and Safety of Tolvaptan in Heart Failure Patients with Volume Overload Despite the Standard Treatment with Conventional Diuretics: A Phase III, Randomized, Double-blind, Placebo-controlled Study (QUEST Study). Cardiovascular Drugs and Therapy 2011; 25:33-45.

[10] Decaux G, Soupart A. Treatment of symptomatic hyponatremia. American Journal of the Medical Sciences 2003; 326: 25-30.

[11] Dahl E, Gluud L L, Kimer N, et al. Meta-analysis: The safety and efficacy of vaptans (tolvaptan, satavaptan and lixivaptan) in cirrhosis with ascites or hyponatraemia. Alimentary Pharmacology \& Therapeutics 2012; 36:619-626.

[12] Yan L, Xie F, Lu J, et al. The treatment of vasopressin V2-receptor antagonists in cirrhosis patients with ascites: a meta-analysis of randomized controlled trials. BMC Gastroenterology 2015; 15: 65(2015).

[13] Bellos I, Kontzoglou K, Psyrri A, et al. Tolvaptan Response Improves Overall Survival in Patients with Refractory Ascites: A Meta-Analysis. Dig Dis 2019; doi: 10.1159/000503559

[14] Guo H, Wu LJ, Jin Z, et al. Vasopressin V2-receptor antagonist tolvaptan for treating cirrhosis patients with hyponatremia and hepatic edema: A systemic review (in Chinese). World Chinese Journal of Digestology 2016; 24:938-946

[15] Higgins JPT, Green S (editors). Cochrane Handbook for Systematic Reviews of Interventions Version 5.1.0. The Cochrane Collaboration. [http://handbook.cochrane. org]. Published March 15, 2011. Accessed August 15, 2019.

[16] Andrés Cárdenas, Pere Ginès, Marotta $P$, et al. Tolvaptan, an oral vasopressin antagonist, in the treatment of hyponatremia in cirrhosis. Journal of Hepatology 2012; 56:571-578.

[17] Isao Sakaida, Seiji Kawazoe, Kozo Kajimura, et al. Tolvaptan for improvement of hepatic edema: A phase 3, multicenter, randomized, double-blind, placebo-controlled trial. Hepatology Research 2014; 44:73-82. 
[18] Kiwamu Okita, Seiji Kawazoe, Chitomi Hasebe, et al. Dose-finding trial of tolvaptan in liver cirrhosis patients with hepatic edema: A randomized, double-blind, placebo-controlled trial. Hepatology Research 2014; 44:83-91.

[19] Haruki Uojima, Hisashi Hidaka, Tsuyoshi Nakayama, et al. Efficacy of combination therapy with natriuretic and aquaretic drugs in cirrhotic ascites patients: A randomized study. World Journal of Gastroenterology 2017; 23:80628072.

[20] Yong Feng Wang, Jie Ting Tang, Tao Han, et al. Tolvaptan in Chinese cirrhotic patients with ascites: A randomized, placebo-controlled phase 2 trial Journal of Digestive Diseases. 2018; 19:144-154.

[21] Wang Shuzhen, Zhang Xin, Han Tao, et al. Tolvaptan treatment improves survival of cirrhotic patients with ascites and hyponatremia. BMC Gastroenterology 2018; 18:137.

[22] Umemura T, Shibata S, Sekiguchi T, et al. Serum sodium concentration is associated with increased risk of mortality in patients with compensated liver cirrhosis. Hepatology Research 2015; 45:739-744.

[23] Kogiso T, Kobayashi M, Yamamoto K, et al. The Outcome of Cirrhotic Patients with Ascites Is Improved by the Normalization of the Serum Sodium Level by Tolvaptan. Internal Medicine 2017; 56:2993-3001.

[24] Heuman D M. Hyponatremia and Mortality among Patients on the Liver-Transplant Waiting List. New England Journal of Medicine 2008; 359:1018-1026.

[25] Chen RP, Zhu Ge XJ, Huang ZM, et al. Prophylactic Use of Transjugular Intrahepatic Portosystemic Shunt Aids in the Treatment of Refractory Ascites. Journal of Clinical Gastroenterology 2014; 48:290-299.

[26] Bhogal HK, Sanyal AJ. Using Transjugular Intrahepatic Portosystemic Shunts for Complications of Cirrhosis. Clinical Gastroenterology and Hepatology 2011; 9:936-946.

\section{Tables}

Table 1: Characteristics of included studies 


\begin{tabular}{|c|c|c|c|c|c|c|c|c|c|c|c|}
\hline \multirow[t]{2}{*}{$y$} & \multirow[t]{2}{*}{ Country } & \multirow[t]{2}{*}{ Multicenter } & \multirow{2}{*}{$\begin{array}{l}\text { Conventional } \\
\text { diuretic }\end{array}$} & \multirow{2}{*}{$\begin{array}{l}\text { Treatment } \\
\text { Duration }\end{array}$} & \multirow[t]{2}{*}{ Treatment } & \multirow[t]{2}{*}{$\mathrm{N}$} & \multirow{2}{*}{$\begin{array}{c}\text { Age } \\
\text { (year) }\end{array}$} & \multirow{2}{*}{$\begin{array}{c}\text { Female } \\
(\mathrm{n}, \%)\end{array}$} & \multicolumn{3}{|c|}{ Child-Pugh Grade (n, \%) } \\
\hline & & & & & & & & & $\mathrm{A}$ & B & $\mathrm{C}$ \\
\hline \multirow[t]{2}{*}{$\mathrm{s}$} & USA & Yes & Yes & $4 \mathrm{~d}$ & $\begin{array}{l}\text { Tolvaptan } \\
15-60 \\
\mathrm{mg} / \mathrm{d}\end{array}$ & 57 & $52 \pm 8$ & $13(22.8)$ & $6(10.5)$ & $28(49.1)$ & $19(33.3)$ \\
\hline & & & & & Placebo & 63 & $55 \pm 9$ & $19(30.2)$ & $11(17.5)$ & $33(52.4)$ & $16(25.4)$ \\
\hline \multirow[t]{6}{*}{$\mathrm{lu}$} & Japan & Yes & Yes & $7 d$ & $\begin{array}{l}\text { Tolvaptan } \\
7.5 \mathrm{mg} / \mathrm{d}\end{array}$ & 25 & $65 \pm 9$ & $7(28.0)$ & $1(4.0 \square$ & $13(52.0)$ & $11(44.0)$ \\
\hline & & & & & $\begin{array}{l}\text { Tolvaptan } \\
15 \mathrm{mg} / \mathrm{d}\end{array}$ & 25 & $65 \pm 10$ & $4(16.0)$ & $0(0)$ & $15(60.0)$ & $10(40.0)$ \\
\hline & & & & & $\begin{array}{l}\text { Tolvaptan } \\
30 \mathrm{mg} / \mathrm{d}\end{array}$ & 25 & $63 \pm 10$ & $10(40.0)$ & $2(8.0)$ & $13(52.0)$ & $10(40.0)$ \\
\hline & & & & & Placebo & 26 & $64 \pm 10$ & $9(34.6)$ & $0(0)$ & $16(61.5)$ & $10(38.5)$ \\
\hline & Japan & Yes & Yes & $7 d$ & $\begin{array}{l}\text { Tolvaptan } \\
7.5 \mathrm{mg} / \mathrm{d}\end{array}$ & 82 & $68(31-80)$ & $30(36.6)$ & $0(0)$ & $44(53.7)$ & $38(46.3)$ \\
\hline & & & & & Placebo & 80 & $69(46-78)$ & $31(38.8)$ & $0(0)$ & $54(67.5)$ & $26(32.5)$ \\
\hline \multirow[t]{7}{*}{$\mathrm{i}$} & Japan & Yes & Yes & $7 d$ & $\begin{array}{l}\text { Tolvaptan } \\
7.5 \mathrm{mg} / \mathrm{d}\end{array}$ & 28 & $69.3 \pm 11.8$ & $11(39.3)$ & $0(0)$ & $16(57.1)$ & $12(42.9)$ \\
\hline & & & & & Placebo & 28 & $69.4 \pm 12.6$ & $13(46.4)$ & $0(0)$ & $15(53.6)$ & $13(46.4)$ \\
\hline & China & Yes & Yes & $7 d$ & $\begin{array}{l}\text { Tolvaptan } \\
15 \mathrm{mg} / \mathrm{d}\end{array}$ & 57 & $49.9 \pm 9.2$ & $11(19.3)$ & $2(3.6)$ & $29(51.8)$ & $25(44.6)$ \\
\hline & & & & & $\begin{array}{l}\text { Tolvaptan } \\
30 \mathrm{mg} / \mathrm{d}\end{array}$ & 63 & $63.0 \pm 10.0$ & $15(23.8)$ & $2(3.2)$ & $40(63.5)$ & $21(33.3)$ \\
\hline & & & & & Placebo & 62 & $48.3 \pm 10.5$ & $18(29.0)$ & $3(4.8)$ & $35(56.5)$ & $24(38.7)$ \\
\hline & China & Yes & Yes & $7 d$ & $\begin{array}{l}\text { Tolvaptan } \\
15-60 \\
\mathrm{mg} / \mathrm{d}\end{array}$ & 169 & $54.6 \pm 8.6$ & $33(19.5)$ & $2(1.2)$ & $113(66.8)$ & $54(32.0)$ \\
\hline & & & & & Placebo & 61 & $51.6 \pm 10.7$ & $12(19.7)$ & $1(1.6)$ & $45(73.8)$ & $15(24.6)$ \\
\hline
\end{tabular}

\section{Appendices}

Supplement Table 1: The detailed Mate analysis results of various common adverse events.

Supplement Fig 1: The risk of bias within studies.

Supplement Fig 2: A: The result of sensitivity analysis for the comparison between the combination diuretic treatment groups and the conventional diuretic treatment groups about the mean change of serum sodium concentration $(\mathrm{mmol} / \mathrm{L})$ after 7-days intervention. B: The result of sensitivity analysis for the comparison between the combination diuretic treatment groups and the conventional diuretic treatment groups about total adverse events.

\section{Figures}




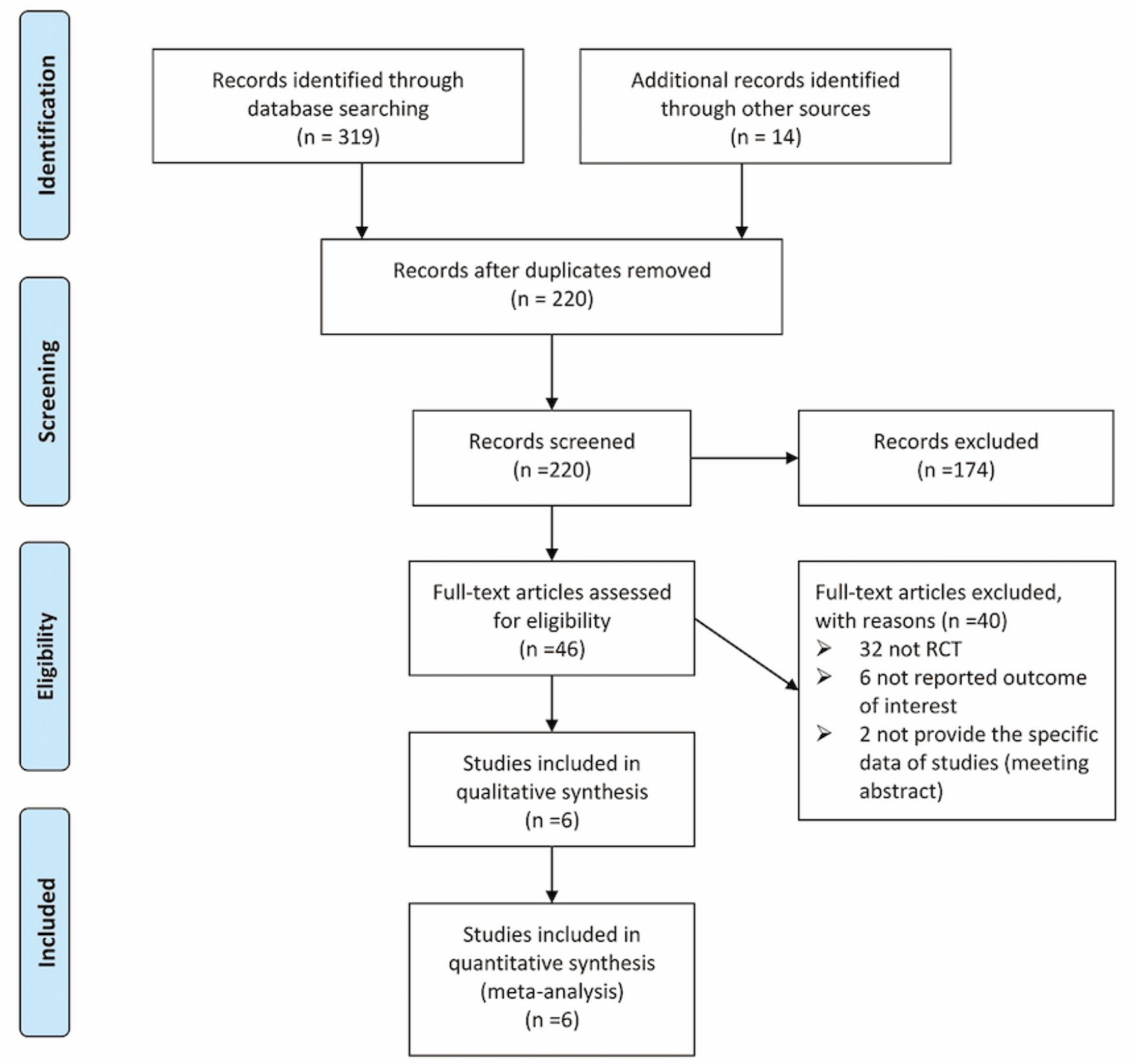

Figure 1

Identification process of the included studies. 


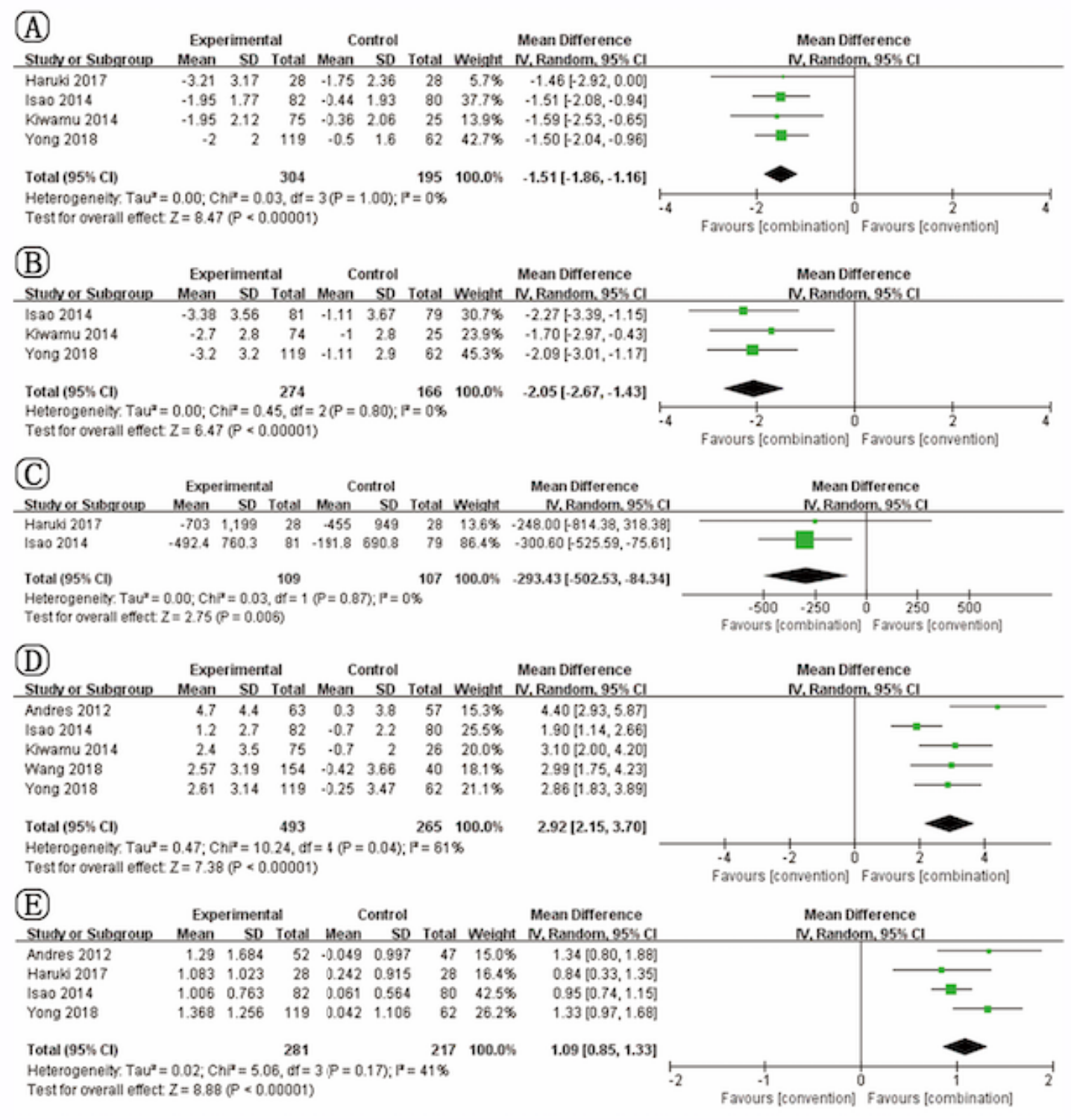

\section{Figure 2}

A: The comparison between the combination diuretic treatment groups and the conventional diuretic treatment groups about the mean change of weight $(\mathrm{Kg})$ after 7-days intervention. B: The comparison between the combination diuretic treatment groups and the conventional diuretic treatment groups about the mean change of abdominal girth $(\mathrm{cm})$ after 7-days intervention. C: The comparison between the combination diuretic treatment groups and the conventional diuretic treatment groups about the mean change of ascites volume $(\mathrm{mL})$ after 7-days intervention. $\mathrm{D}$ : The comparison between the combination diuretic treatment groups and the conventional diuretic treatment groups about the mean change of serum sodium concentration (mmol/L) after 7-days intervention. E: The comparison between the combination diuretic treatment groups and the conventional diuretic treatment groups about the mean change of urine volume $(\mathrm{L})$ on Day 1 of intervention. 


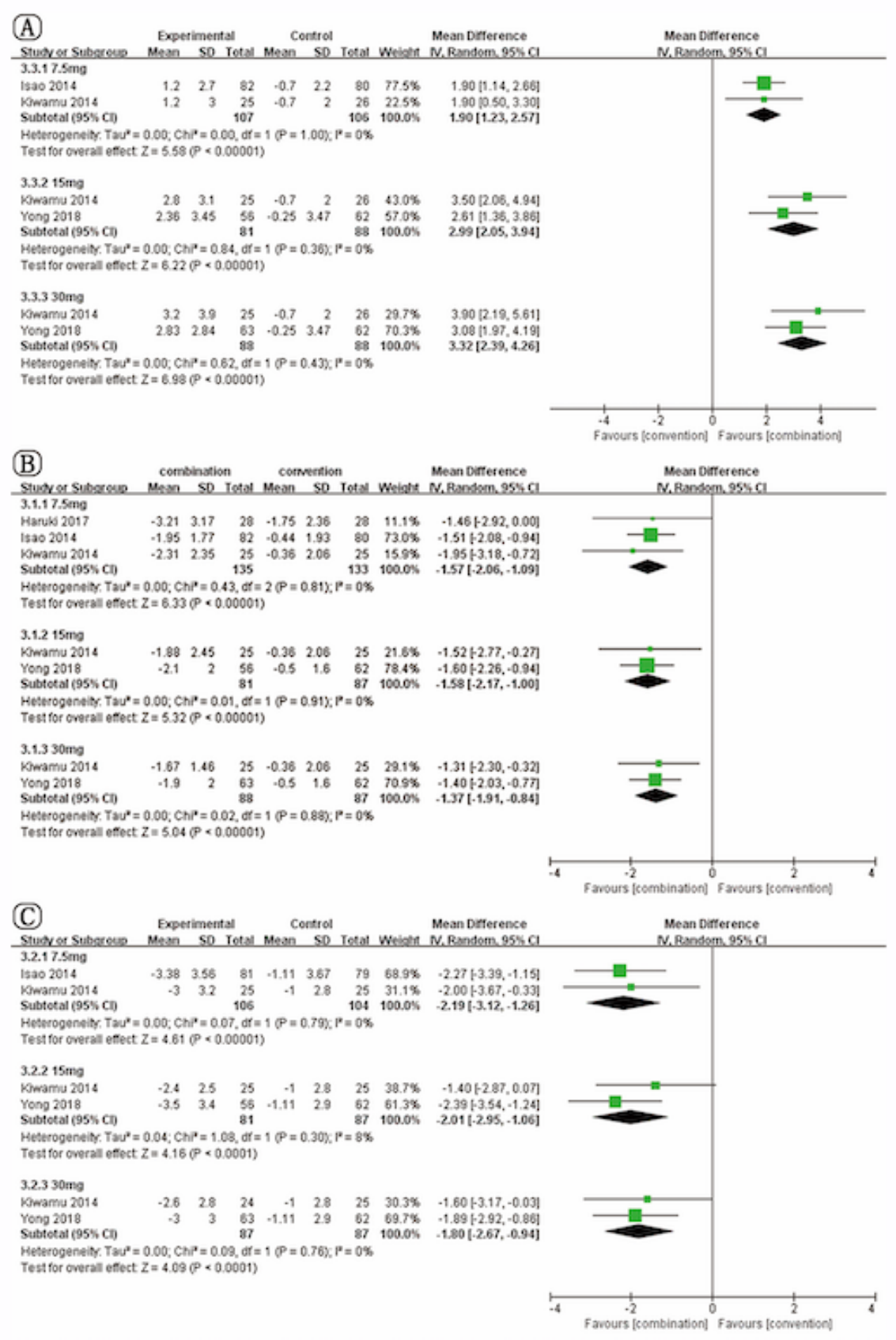

\section{Figure 3}

A: The comparison between the combination diuretic treatment groups and the conventional diuretic treatment groups about the mean change of serum sodium concentration $(\mathrm{mmol} / \mathrm{L})$ after 7-days intervention for the different daily dose of Tolvaptan (7.5mg-15mg-30mg). B: The comparison between the combination diuretic treatment groups and the conventional diuretic treatment groups about the mean change of weight $(\mathrm{Kg})$ after 7-days intervention for the different daily dose of Tolvaptan (7.5mg-15mg-30mg). C: The comparison between the combination diuretic treatment groups and the conventional diuretic treatment groups about the mean change of abdominal girth $(\mathrm{cm})$ after 7-days intervention for the different daily dose of Tolvaptan (7.5mg-15mg-30mg). 


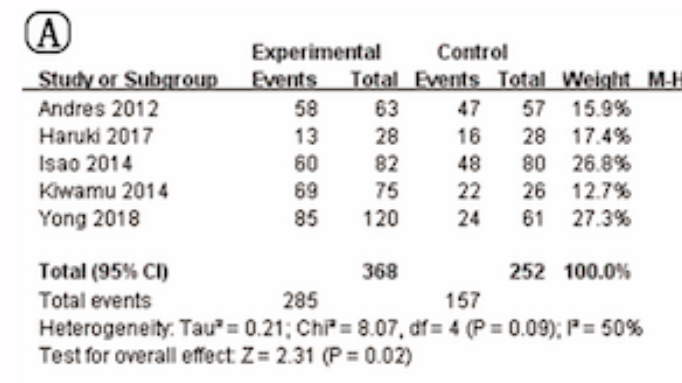

Odds Ratio Odds Ratio

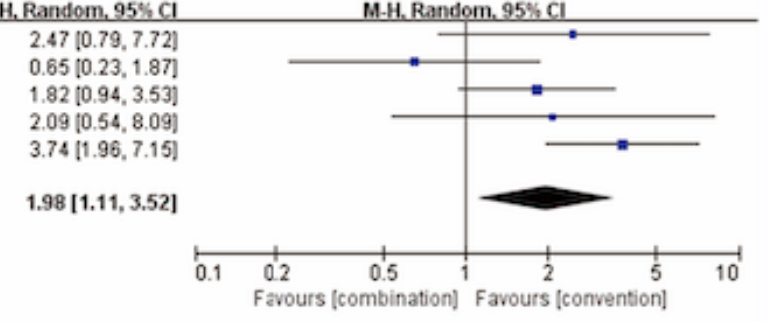

\begin{tabular}{|c|c|c|c|c|c|}
\hline & \multicolumn{2}{|c|}{ Experimental } & \multicolumn{2}{|c|}{ Control } & \multirow[b]{2}{*}{ Weight } \\
\hline Study or Substoup & Events & Total & Events & Total & \\
\hline Andres 2012 & 24 & 63 & 17 & 57 & $54.7 \%$ \\
\hline Haruki 2017 & 0 & 28 & 2 & 28 & $3.3 \%$ \\
\hline Isao 2014 & 7 & 82 & 10 & 80 & $30.6 \%$ \\
\hline Yong 2018 & 5 & 120 & 2 & 61 & $11.4 \%$ \\
\hline Total $(95 \% \mathrm{Cl})$ & & 293 & & 226 & $100.0 \%$ \\
\hline Total events & 36 & & 31 & & \\
\hline $\begin{array}{l}\text { Heterogeneity. Tauª } \\
\text { Test for overall effec }\end{array}$ & $\begin{array}{l}0.00 ; C h i \\
Z=0.15\end{array}$ & $\begin{array}{l}=2.79, \\
=0.88\end{array}$ & $d f=3(P$ & $=0.43$ & $P=0 \%$ \\
\hline
\end{tabular}
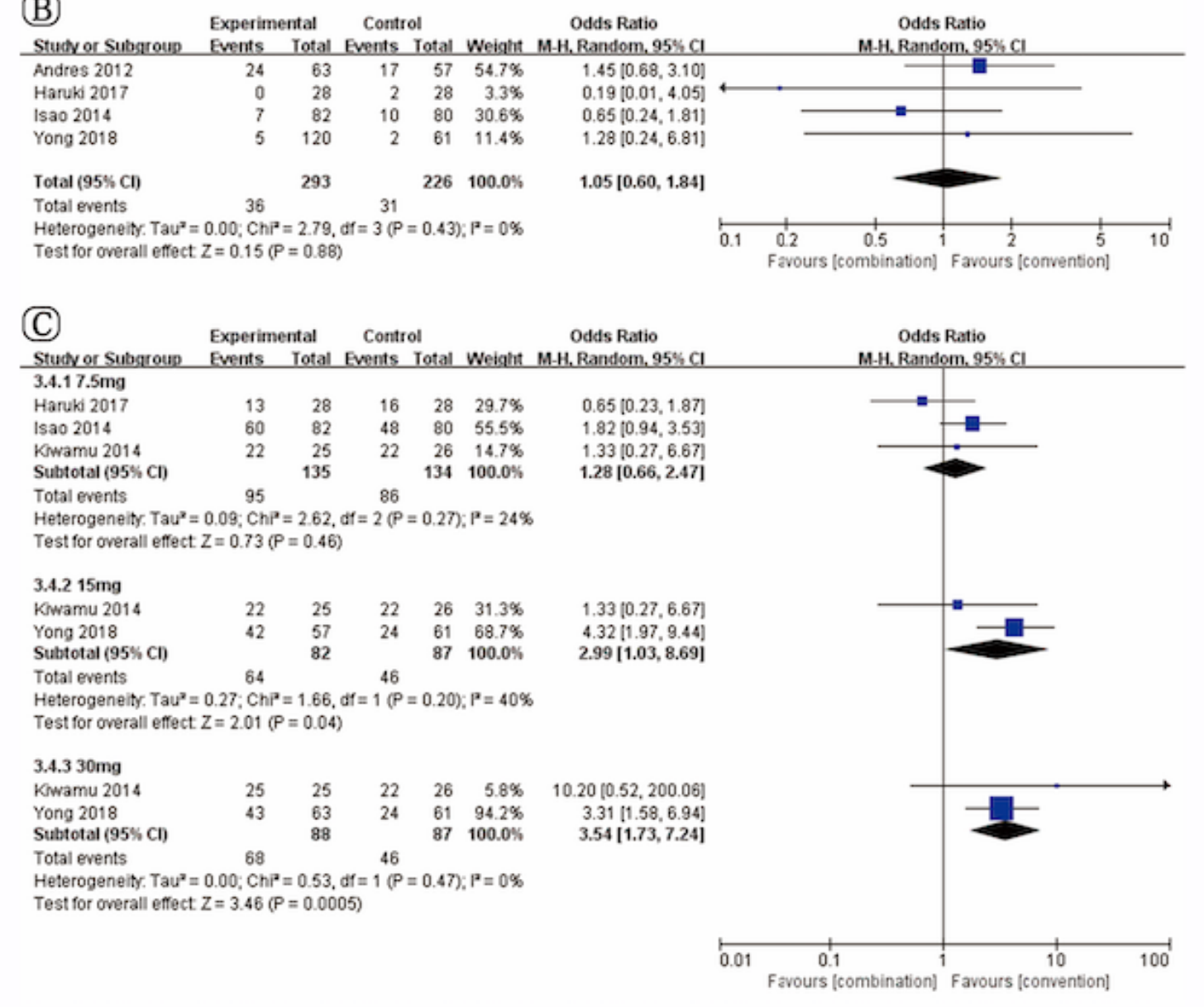

\section{Figure 4}

$\mathrm{A}$ : The comparison between the combination diuretic treatment groups and the conventional diuretic treatment groups about total adverse events. B: The comparison between the combination diuretic treatment groups and the conventional diuretic treatment groups about serious adverse events. C: The comparison between the combination diuretic treatment groups and the conventional diuretic treatment groups about total adverse events with the different dose of tolvaptan. 


\section{(A)}

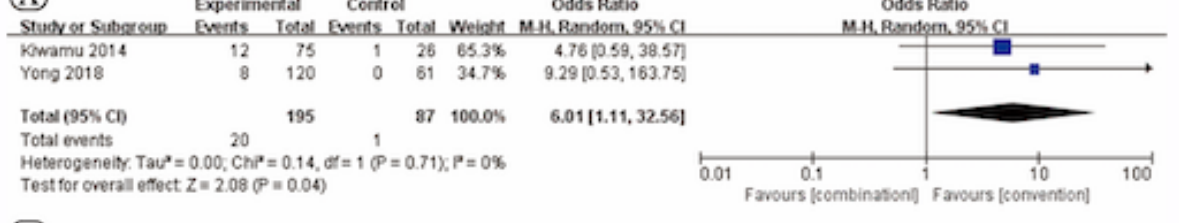

(B)

Experimental Control

Odds Ratio

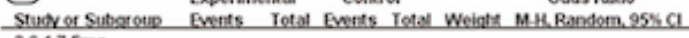
3.6.17.5mg Haruk 2017 Isao 2014 kewamu 2014 Subtetat (95s CI) Total ewents Heterogeneir, $\operatorname{Tau}^{2}=4.73 ; \mathrm{ChP}=11.27$, of $=2(\mathrm{P}=0.004) ; P=82 \%$

Test for overall ettect $Z=0.20(P=0.84)$

$3.6 .215 \mathrm{mg}$

Keramu 2014

Kowamu 2014

Yong 2018 (cototal $(95 \% \mathrm{Cl})$

Total events

Heterogeneity, Tau $^{*}=0.62 ;$ ChP $=1.80$, df $=1$ ( $\left.P=0.18\right) ; P=44 \%$

Test for owerall efect $Z=2.99(P=0.003)$

$3.6 .330 \mathrm{mg}$

Kewamu 2014

Yong 2018

Subtotal $(95 \mathrm{SC})$

Total events

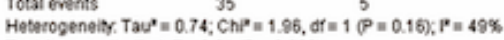

Test for overall effect $Z=3.01(P=0.003)$

Odds Ratio MH. Random. 955 C

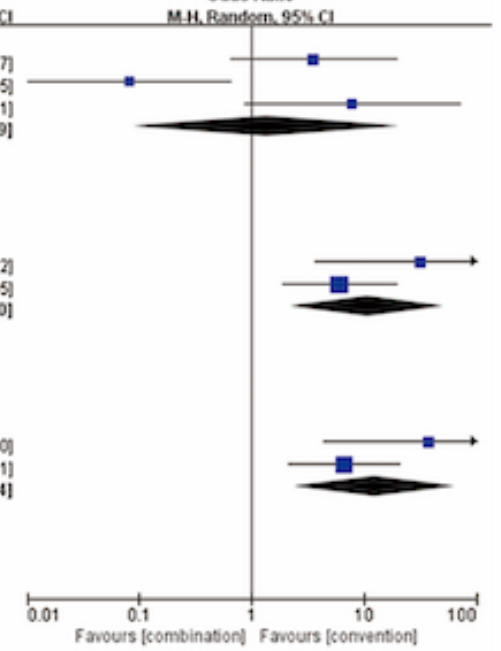

(C)

Experimental Control

Odds Patio

3.7.17.5mg

Harubs 2017

lisao 2014

Kisterotat $1955 \mathrm{~cm}$

Total ements

Hetronents

ChP $=8.00$, ef $=2(P=0.02): P=75 \%$

Test for overall effect $Z=0.33$ Q $=0.74$

$3.7 .215 \mathrm{mg}$

19ramu 2014

Yone 2018

Sublotal (95s) Ci)

Total ewonts

Heterogeneity, Tau $=0.00 ;$ ChP $=0.14$, ef $=1$ P $P=0.71), P=0$.

Test for overall effect $Z=2.48(p=0.01)$

$3.7 .330 \mathrm{mg}$

Kewamu 2014

Yong 2018

Sutototal $(95 \mathrm{sCl})$

Total ewents

Heterogeneity, Tau" $=0.00 ; \mathrm{ChP}^{2}=0.65$, eff $=1(P=0.42), P=0 \%$

Test for overall effect $Z=3.38(p=0.0007)$
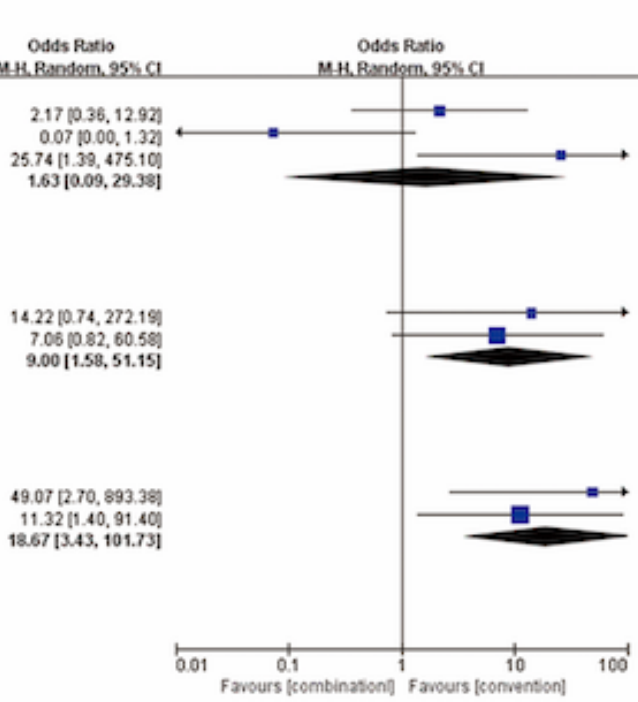

\section{Figure 5}

A: The comparison between the combination diuretic treatment groups and the conventional diuretic treatment groups about blood uric acid increasing as an adverse event. B: The comparison between the combination diuretic treatment groups and the conventional diuretic treatment groups about thirst as an adverse event with the different dose of tolvaptan. C: The comparison between the combination diuretic treatment groups and the conventional diuretic treatment groups about pollakiuria as an adverse event with the different dose of tolvaptan.

\section{Supplementary Files}

This is a list of supplementary files associated with this preprint. Click to download. 
- Availabilityofdata.xlsx

- Supplement.docx

- PRISMA2009checklist.doc 